\title{
Multidisciplinary pain management program for patients with chronic musculoskeletal pain in Japan: a cohort study
}

\author{
This article was published in the following Dove Press journal:
}

Journal of Pain Research

\author{
Naoto Takahashi ${ }^{1,2}$ \\ Kozue Takatsuki ${ }^{1}$ \\ Satoshi Kasahara ${ }^{1,2}$ \\ Shoji Yabuki ${ }^{1,2}$ \\ 'Department of Pain Medicine, \\ Fukushima Medical University School of \\ Medicine, Fukushima 9601295, Japan; \\ ${ }^{2}$ Pain Management Center, Hoshi General \\ Hospital, Koriyama 963-850I, Japan
}

\begin{abstract}
Introduction: Multidisciplinary pain management is a useful method for treating chronic musculoskeletal pain. Currently, few facilities in Japan offer multidisciplinary pain treatment, especially in the inpatient setting. We implemented a multidisciplinary pain management program based on International Association for the Study of Pain recommendations. This study described our initial efforts in implementing the program, and reported 3- and 6-month follow-up results.
\end{abstract}

Materials and methods: Our pain management team included orthopedic surgeons, psychiatrists, nurses, physical therapists, clinical psychologists, pharmacists, and nutritionists. The 3-week inpatient pain management program comprised exercise therapy, psychotherapy, and patient education. We evaluated patients using the Brief Pain Inventory (BPI), Pain Catastrophizing Scale (PCS), Pain Disability-Assessment Scale (PDAS), Hospital Anxiety and Depression Scale (HADS), Pain Self-Efficacy Questionnaire (PSEQ), EuroQol Five Dimensions (EQ-5D), and physical examinations (flexibility, muscle endurance, walking ability, and physical fitness). Statistical analyses were performed using paired $t$-tests and Wilcoxon matched-pairs signed-rank sum tests with Bonferroni correction after the Friedman test.

Results: Data for 23 patients were analyzed before and immediately after the program. Statistically significant improvements were seen in BPI, PCS, PDAS, HADS, PSEQ, EQ-5D, flexibility, muscle endurance, walking ability, and physical fitness. Eight patients were also assessed 3 and 6 months after the program. PCS (rumination and helplessness) scores and flexibility showed significant improvement at 3 and 6 months. Significant improvement was seen in PDAS and HADS (anxiety) scores and muscle endurance at 6 months, and in PSEQ scores immediately and at 3 and 6 months.

Conclusion: Our inpatient pain management program can improve patients' physical function and ability to cope with chronic musculoskeletal pain, which supports improved quality of life. Our program is currently being expanded to better assist patients with chronic musculoskeletal pain.

Keywords: chronic musculoskeletal pain, multidisciplinary pain treatment, biopsychosocial model, inpatient pain management program

\section{Plain language summary}

Multidisciplinary pain management is a useful method for treating chronic musculoskeletal pain. Currently, few facilities in Japan offer multidisciplinary pain treatment, especially in the inpatient setting. We implemented a multidisciplinary pain management program based on International Association for the Study of Pain recommendations. This study described our initial efforts in implementing the program and reported 3- and
Department of Pain Medicine, Fukushima Medical University School of Medicine, I Hikarigaoka, Fukushima 960-1295, Japan

Tel +8I 245471964

Fax +8I 245471966

Email naoto-t@fmu.ac.jp 
6-month follow-up results. Our pain management team included orthopedic surgeons, psychiatrists, nurses, physical therapists, clinical psychologists, pharmacists, and alnutritionists. The 3-week inpatient pain management program involved exercise therapy, psychotherapy, and patient education. We evaluated patients using the Brief Pain Inventory (BPI), Pain Catastrophizing Scale (PCS), Pain Disability-Assessment Scale (PDAS), Hospital Anxiety and Depression Scale (HADS), Pain Self-Efficacy Questionnaire (PSEQ), EuroQol Five Dimensions (EQ-5D), and physical examinations (flexibility, muscle endurance, walking ability, and physical fitness). We analyzed results for 23 patients before and immediately after the program. There were statistically significant improvements in all measures immediately after the program. Eight patients were also assessed 3 and 6 months after the program. PCS (rumination and helplessness) scores and flexibility showed significant improvement at 3 and 6 months. Significant improvement was seen in PDAS and HADS (anxiety) scores and muscle endurance at 6 months, and in PSEQ scores immediately and at 3 and 6 months. Our inpatient pain management program improves patients' physical function and ability to cope with chronic musculoskeletal pain, supporting improved quality of life. Our program is currently being expanded to better assist patients with chronic musculoskeletal pain.

\section{Introduction}

Musculoskeletal pain is a common problem that affects millions of people worldwide. Chronic musculoskeletal pain reduces people's quality of life (QOL) and may hinder normal social life. A multidisciplinary approach for pain management is useful for treating chronic musculoskeletal pain, and has been used in the USA since the 1950s. A biopsychosocial model $^{1}$ of well-being is an important concept in multidisciplinary pain management. This is a general model or approach based on the assumption that biological, psychological, and social factors play significant roles in affecting human function during disease or illness. Multidisciplinary pain treatment requires special facilities. However, few facilities in Japan are able to provide multidisciplinary pain treatment, particularly in an inpatient setting. We implemented an inpatient multidisciplinary pain management program in our hospital $^{2}$ based on the biopsychosocial model recommended by the International Association for the Study of Pain (IASP). ${ }^{3-7}$ We previously reported our inpatient multidisciplinary pain management program was being expanded to improve support for patients with chronic musculoskeletal pain. ${ }^{2}$ However, our previous results reflected an evaluation immediately after the program. Therefore, it was unclear whether our inpatient multidisciplinary pain management program could lead to long-term improvement for patients with chronic musculoskeletal pain.

This article aimed to describe our initial efforts in implementing the multidisciplinary pain management program for Japanese patients, which used a biopsychosocial model for pain self-management. We also aimed to report results from the 3- and 6-month follow-up.

\section{Materials and methods}

\section{Patients}

We treated 102 patients with chronic musculoskeletal pain from April 2015 to March 2018. Twenty-three patients completed our inpatient multidisciplinary pain management program. Data for these 23 patients were analyzed before and immediately after the program. Eight patients were also able to be assessed at 3 and 6 months after the program. The remaining 15 patients dropped out of this study. Two patients recovered completely and did not need to continue regular hospital visits, eight patients lived far from our hospital and could not continue regular hospital visits, four patients did not agree to regular hospital visits, and one patient had developed lung cancer.

\section{Multidisciplinary pain management program}

The pain management center at Hoshi General Hospital has a team of orthopedic surgeons, psychiatrists, nurses, physical therapists, clinical psychologists, pharmacists, and nutritionists. The center was established in April 2015 and is technically supported by the Department of Pain Medicine at Fukushima Medical University School of Medicine. First, patients are screened by orthopedic surgeons and physical therapists for mechanical and biological changes in the body. Psychiatrists and clinical psychologists then evaluate patients' psychosocial and social factors, and radiologists assess their cortical function. A flowchart of our multidisciplinary pain treatment system is shown in Figure 1. The inclusion criteria for our 3-week inpatient multidisciplinary pain management program were: (1) patients who had difficulty working or attending school because of chronic musculoskeletal pain, (2) patients confined to life at home but that wished 
to return to work or school, (3) patients who accepted our program, and (4) patients who were able to pay the hospital expenses for our inpatient program. Exclusion criteria were: (1) older adults who were unable to answer the questionnaires; (2) patients with dementia or intellectual disabilities; and (3) patients who would have difficulty participating in the program (determined by multidisciplinary conference).

The program incorporates exercise therapy, psychotherapy, and patient education. Specific details of the program are as follows. (1) Exercise therapy, which comprises physical fitness and individual training by physical therapists, including aerobic exercise (walking, underwater exercise) and strengthening and stretching of muscles. A sit-up exercise was used to strengthen trunk flexor muscles and an extension exercise was used to strengthen trunk extensor muscles. Exercise therapy took place over 6$7 \mathrm{hrs} /$ week, giving a total of $20 \mathrm{hrs}$. (2) Psychotherapy and cognitive behavioral therapy were used to develop patients' assertiveness (three 60-120 min sessions). Patients also received relaxation training (two 60120 min sessions), and role-playing to increase healthy behaviors and decrease pain behavior (two 60-120 min sessions). All sessions were led by psychologists. (3) Patient education, which was delivered by various specialists. These sessions covered pain mechanisms (orthopedic surgeon), exercise and pacing (physical therapist), assertiveness and relaxation training (psychologist), side effects of drugs (pharmacist) and daily life habits associated with nutrition (nutritionist). Each education session lasted 3060 mins, and patients received 20 sessions in total.

The program aims to help patients return to a functional daily life by acquiring new habits and coping methods for their pain. A characteristic of our program is that family members or significant others also participated in some education and psychotherapy sessions. ${ }^{7}$ Our previous report $^{2}$ detailed preliminary results for our 3-week inpatient multidisciplinary pain management program.

\section{Specialists' roles}

The roles of each of the seven specialist groups have been described in detail in our previous report. ${ }^{3}$ An additional role performed by clinical psychologists was classifying patients into three subgroups: dysfunctional (DYS-type), interpersonally distressed (ID-type), and adaptive coper (AC-type) using the West Haven-Yale Multidimensional Pain Inventory (WHYMPI). ${ }^{8}$ The WHYMPI is a multidimensional assessment instrument for patients with chronic pain. These three subgroups have specific symptom patterns that could be evaluated by cluster analysis of the nine main WHYMPI scales. Characteristics of the DYS-type subgroup were high pain severity, low function, high depression, and low life control. The ID-type subgroup was characterized by a low level of support from their partner and social environment. Finally, characteristics of the AC-type subgroup were relatively low pain

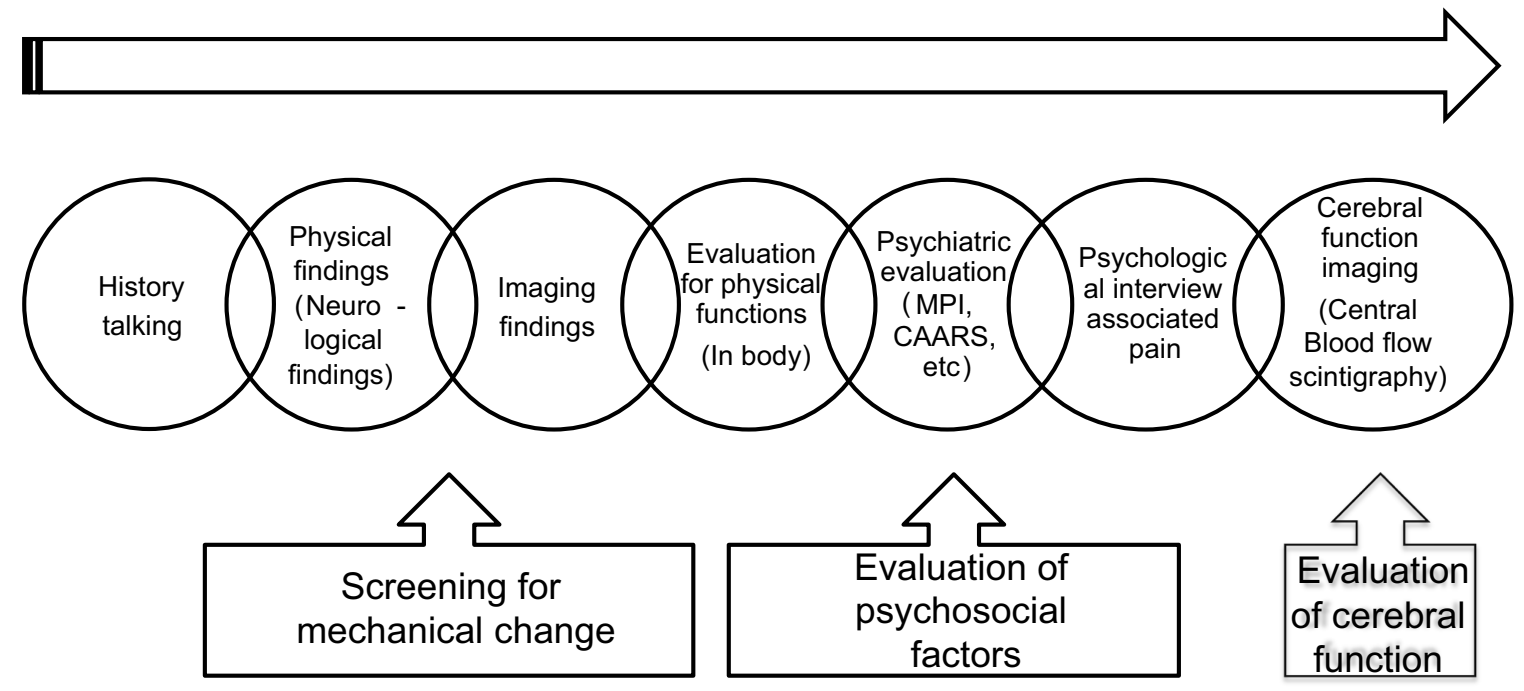

Figure I Flowchart for the evaluation and diagnosis of chronic musculoskeletal pain at Hoshi General Hospital. First, orthopedic surgeons evaluate physical, neurological, and imaging findings and diagnose the presence of any specific musculoskeletal disorders. Second, physical therapists evaluate physical function. Third, psychiatrists diagnose any psychological disorders associated with chronic musculoskeletal pain, including psychiatric disorders. Fourth, clinical psychologists evaluate the psychosocial factors associated with pain. Finally, we perform brain magnetic resonance imaging and cerebral blood-flow scintigraphy to evaluate cortical function.

Abbreviations: MPI, Multidimensional Pain Inventory; CAARS, Conners' Adult ADHD Rating Scales. 
intensity and relatively low interference from pain (functional interference). We classified patients into these subgroups because subgroups were assigned during initial pain psychology interviews, and pain self-management was taught depending on subgroup assignment.

\section{Evaluations of pain, associated factors, and physical function}

We evaluated inpatients with chronic musculoskeletal pain using the Brief Pain Inventory (BPI) ${ }^{9}$ to assess pain severity, and several other scales to assess pain-related psychosocial factors. These scales included the Pain Catastrophizing Scale (PCS) to evaluate the degree of rumination, magnification, and helplessness; ${ }^{10}$ the Pain Disability Assessment Scale (PDAS); ${ }^{11}$ the Hospital Anxiety and Depression Scale (HADS) to assess anxiety and depression; ${ }^{12}$ and the Pain Self-Efficacy Questionnaire (PSEQ). ${ }^{13}$ We also administered the EuroQol five dimensions questionnaire (EQ-5D) ${ }^{14}$ to evaluate patients' QOL. Patients' physical function was measured using the fingerfloor-distance test to evaluate the degree of flexibility, the 30 -second sit-to-stand test to assess muscle endurance, the 2-step test to evaluate walking ability, and 6 mins of walking to assess physical fitness.

\section{Statistical analysis}

We compared patients' pain, associated factors, and physical abilities before and immediately after participation in the 3-week inpatient program. Statistical analyses for each item were performed using paired $t$-tests. We evaluated the same measures at 3 and 6 months after completion of the program. Statistical analyses were performed using Wilcoxon matched-pairs signed-rank sum test with Bonferroni correction after the Friedman test. We considered $p$-values less than 0.05 to be statistically significant. Statistical analyses were performed using IBM SPSS version 25 (IBM Corp., Armonk, NY, USA).

\section{Ethics approval and consent to participate}

This study was approved by the ethics committees of the participating institutions: Fukushima Medical University (Reference number: 2429) and Hoshi General Hospital (Reference number: 27-3). All patients provided written informed consent before this study started.

This study was conducted in accordance with the Declaration of Helsinki.

\section{Results}

In total, 23 patients (eight males, 15 females) aged 20 79 years (mean age 52.2 years) participated in our impatient program from April 2015 to March 2018. Participants' clinical characteristics, chief complaints, structural disorders, and psychiatric diagnoses are shown in Table 1. Analysis of psychiatric diagnoses showed that six patients (one male, five females) had personality disorders and 15 patients (seven males, eight females) had pervasive developmental disorder, attention deficit hyperactivity disorder, or somatoform disorders (Table 1). Only five cases (all females) were without a personality or developmental disorder diagnosis (Table 1). Eighteen patients (78\%) (six males, 12 females) showed abnormal findings on cerebral blood flow scintigraphy.

\section{Results before and immediately after the program (23 patients)}

A comparison of results for the 23 patients before and immediately after the program showed statistically significant improvements in pain and associated factors (Table 2) and physical functions (Table 3).

\section{Results before, immediately after, and 3 and 6 months after the program (eight patients)}

Eight patients (four males, four females) aged 20-69 years (mean age 42.9 years) were also evaluated at 3 and 6 months after the program (marked by bold font in Table 1). Statistically significant improvement was seen in pain and associated factors (Figure 2) and physical functions (Figure 3).

\section{Discussion}

Our inpatient multidisciplinary pain management program was based on a biopsychosocial model guided by IASP recommendations. The present study showed that pain intensity (measured with the BPI) and pain-associated elements including psychosocial factors (measured with the PCS, PDAS, HADS, and PSEQ), QOL (measured with the EQ-5D), flexibility, muscle endurance, and physical fitness were significantly improved after completing the program. In addition, pain relief and improvement in psychosocial factors (PCS rumination and helplessness, PDAS, HADS-Anxiety, and PSEQ) and physical functioning (flexibility and muscle endurance) were maintained for at least 6 months after completing the program. There were minimal detectable changes for pain-associated 


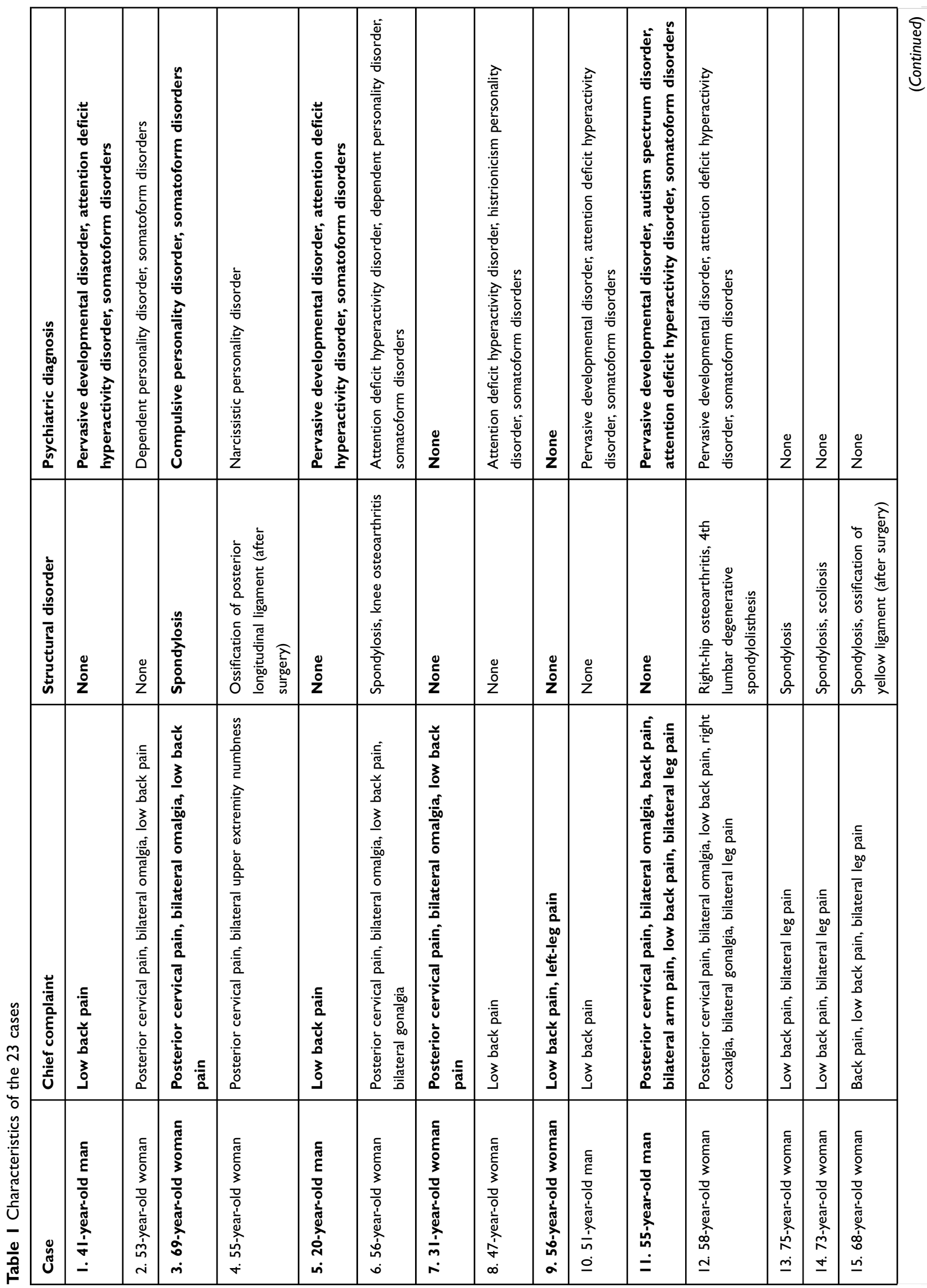




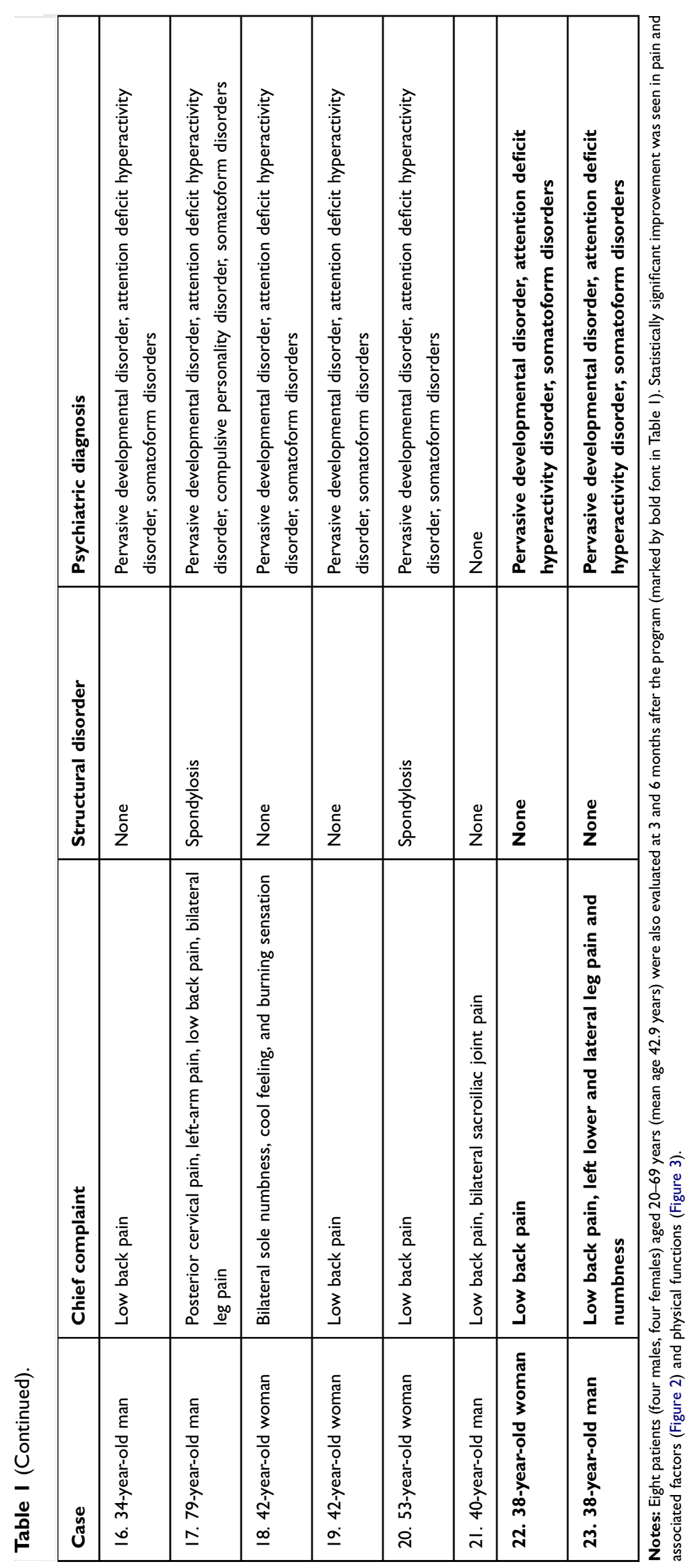


Table 2 Changes in pain and associated factors $(n=23)$

\begin{tabular}{|l|l|l|l|l|}
\hline & $\begin{array}{l}\text { Before program } \\
\text { (mean } \pm \text { SD) }\end{array}$ & $\begin{array}{l}\text { Immediately after program } \\
\text { (mean } \pm \text { SD) }\end{array}$ & $p$-value \\
\hline BPI & $24.5 \pm 10.15$ & $20.0 \pm 8.90$ & 0.0001 & 0.73 \\
PCS (rumination) & $15.1 \pm 5.19$ & $12.7 \pm 5.21$ & 0.004 & 0.001 \\
PCS (magnification) & $6.3 \pm 4.09$ & $4.4 \pm 3.53$ & 0.0001 & 0.67 \\
PCS (helplessness) & $11.5 \pm 5.58$ & $7.5 \pm 5.67$ & 0.0001 & 0.68 \\
PCS (total) & $32.7 \pm 14.05$ & $24.5 \pm 13.68$ & 0.001 & 0.71 \\
PDAS & $29.4 \pm 12.29$ & $18.8 \pm 12.56$ & 0.0001 & 0.64 \\
HADS (anxiety) & $8.7 \pm 4.86$ & $6.2 \pm 4.02$ & 0.001 & 0.66 \\
HADS (depression) & $9.8 \pm 4.81$ & $6.5 \pm 4.35$ & 0.0001 & 0.65 \\
PSEQ & $22.1 \pm 11.90$ & $33.2 \pm 13.93$ & 0.009 \\
EQ-5D & $0.533 \pm 0.1552$ & $0.641 \pm 0.1745$ & 0.69 \\
\hline
\end{tabular}

Notes: $p$-values less than 0.05 were considered statistically significant. $r$ shows the effect size between before and immediately after the program. The cut-off value for the PCS total score is 30 points. The PCS total score was $>30$ points before the program and $<30$ points immediately after program. The result suggested the PCS total score improved more than the minimal detectable change. The PDAS cut-off value is 10 points. The PDAS was $>10$ points before and immediately after program. The result suggested PDAS scores might not have improved to normal condition; however, the PDAS showed significant improvement immediately after the program. For the HADS, 8-10 points indicates suspicion of anxiety/depression and >II points indicates anxiety/depression. The result suggested HADS scores improved more than the minimal detectable change. The minimal clinically important difference for the PSEQ is $>9 \%$. The result suggested PSEQ scores improved more than the minimal detectable change. The BPI and EQ-5D have no clear standards; however, these results suggested pain severity (BPI) and quality of life (EQ-5D) improved immediately after the program. Abbreviations: SD, standard deviation; BPI, Brief Pain Inventory; PCS, Pain Catastrophizing Scale; PDAS, Pain Disability Assessment Scale; HADS, Hospital Anxiety and Depression Scale; PSEQ, Pain Self-Efficacy Questionnaire; EQ-5D, EuroQol Five Dimensions Questionnaire.

Table 3 Changes in physical functions $(n=23)$

\begin{tabular}{|l|l|l|l|l|}
\hline & $\begin{array}{l}\text { Before program } \\
\text { (mean } \pm \text { SD) }\end{array}$ & $\begin{array}{l}\text { Immediately after program } \\
\text { (mean } \pm \text { SD) }\end{array}$ & $\boldsymbol{p}$-value \\
\hline Finger floor distance (flexibility) & $15.8 \pm 16.30$ & $8.1 \pm 13.73$ & 0.005 & 0.59 \\
30-second sit-to-stand test (muscle endurance) & $14.0 \pm 7.46$ & $18.9 \pm 8.87$ & 0.0001 & 0.77 \\
2-step test (walking ability) & $1.3 \pm 0.27$ & $1.4 \pm 0.23$ & 0.01 & 0.53 \\
6 mins walking (physical fitness) & $424.9 \pm 127.64$ & $477.0 \pm 133.96$ & 0.005 & 0.59 \\
\hline
\end{tabular}

Notes: $p$-values less than 0.05 were considered statistically significant. $r$ shows the effect size between before and immediately after the program.

Abbreviation: SD, standard deviation.

elements (Table 2). PCS total scores ranged from 32.724.5 points. The cut-off value for the PCS total score is 30 points. Among our participants, the PCS total score was $>30$ points before program and $<30$ points immediately after program, which showed more improvement in PCS total score than the minimal detectable change. PDAS scores ranged from 29.4-18.8 points. The cut-off value for the PDAS is 10 points; the PDAS score was $>10$ points before and immediately after the program in our participants, which suggested the PDAS was not improved to normal condition in the program. However, participants' PDAS scores still showed significant improvement immediately after the program. HADS scores of $8-10$ points indicate suspicion of anxiety/depression and scores $>11$ points are considered to indicate the presence of anxiety/ depression. $^{13}$ In this study, participants' HADS scores improved more than the minimal detectable change. The minimal clinically important difference for the PSEQ was more than $9 \%,{ }^{15}$ which suggested participants' PSEQ scores improved more than the minimal detectable change. Finally, the BPI and EQ-5D have no clear standards; however, our results suggested that pain severity (BPI) and QOL (EQ-5D) were improved immediately after the program.

\section{Multidisciplinary pain treatment for chronic musculoskeletal pain}

The pathology of chronic musculoskeletal pain has intricately related biological and psychosocial components. As the duration of musculoskeletal pain is lengthened, the casual relationship between pain and pain-related factors becomes obscure. Furthermore, pain behavior and social factors related to pain may become more involved. The IASP indicates that changes in physical and psychosocial 

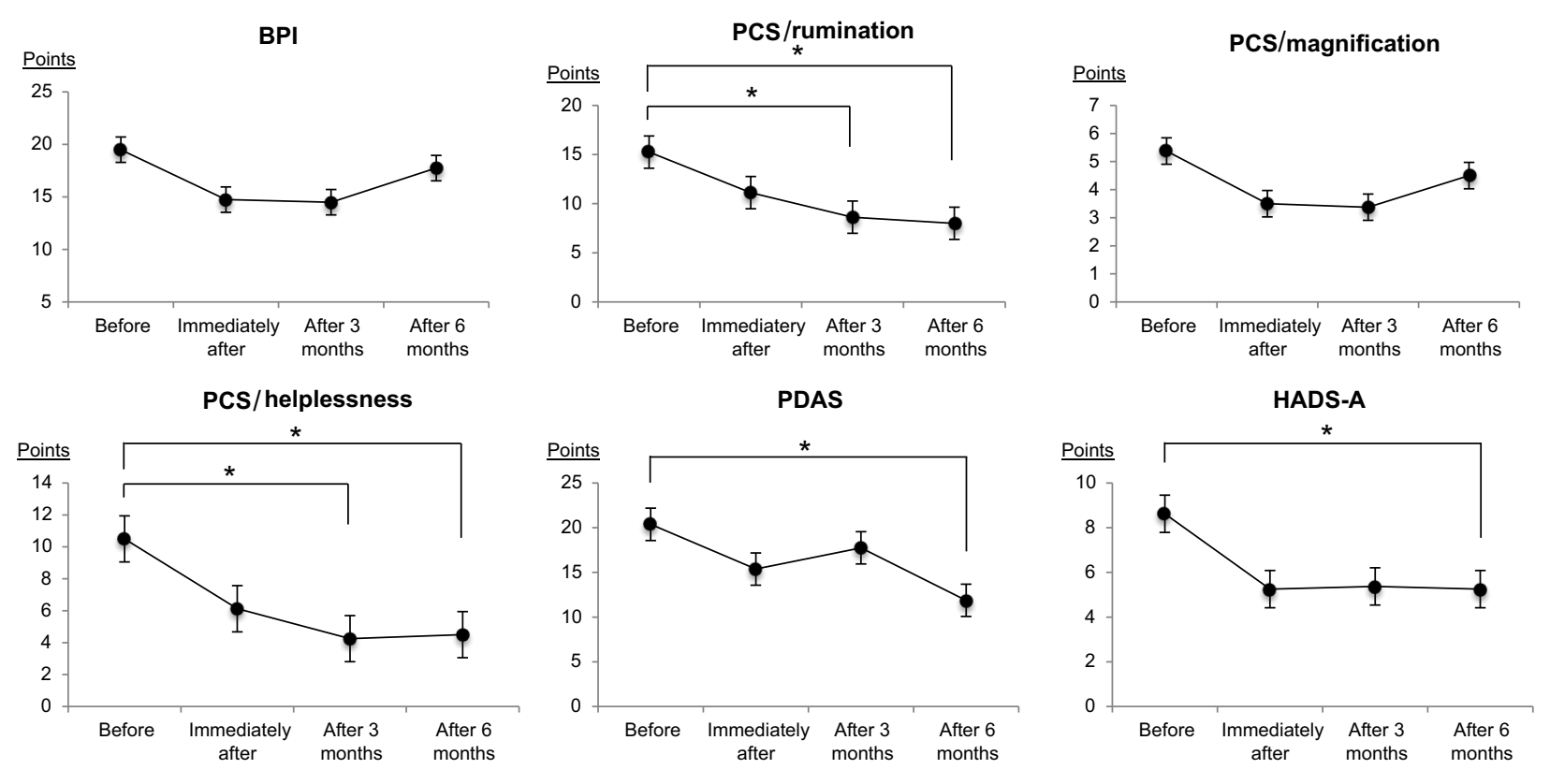

HADS-D
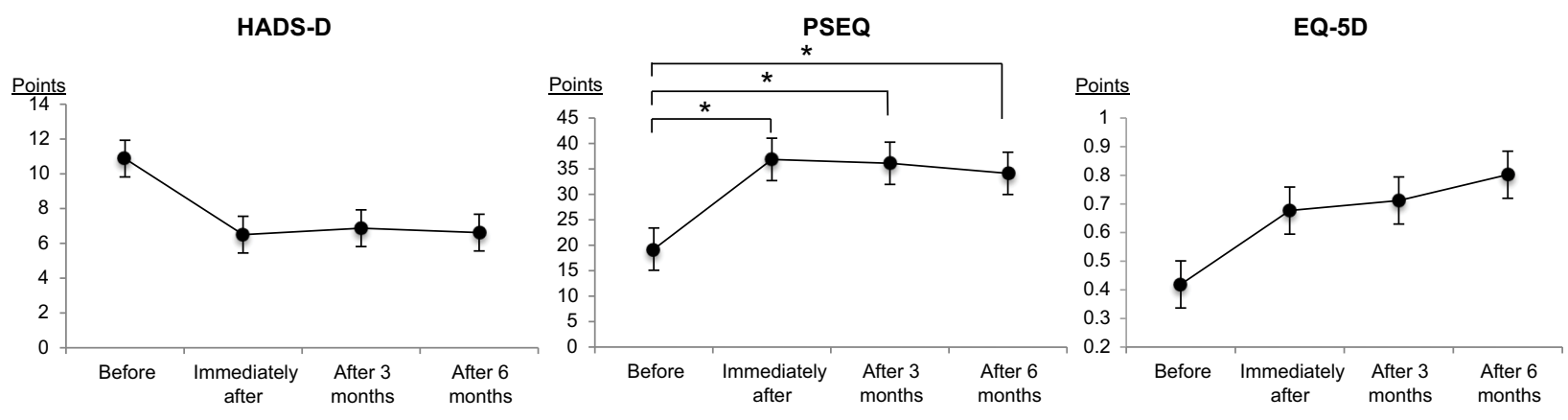

Figure 2 Changes in pain-associated factors. Statistically significant improvement was seen in: PCS (rumination) at $3(p=0.03)$ and 6 ( $p=0.003)$ months after the program; PCS (helplessness) at $3(p=0.012)$ and $6(p=0.04)$ months; PDAS at 6 months $(p=0.04)$; HADS (anxiety) at 6 months $(p=0.016)$; and PSEQ immediately after ( $p=0.004)$, at 3 months $(p=0.012)$, and at 6 months $(p=0.03)$. $* p<0.05$.

Abbreviations: BPI, Brief Pain Inventory; PCS, Pain Catastrophizing Scale; PDAS, Pain Disability Assessment Scale; HADS, Hospital Anxiety and Depression Scale; PSEQ, Pain Self-Efficacy Questionnaire; EQ-5D, EuroQol Five Dimensions Questionnaire.

factors from chronic musculoskeletal pain include muscle weakness associated with reduction in daily activity, somnipathy, malnutrition, drug dependence, dependence on family, isolation from family or society, decline in job performance, and economic burden. These factors may prolong pain duration and decrease the effects of therapy. Therefore, usual treatment delivered by a single department may not be sufficient for people with chronic pain. The IASP recommends multidisciplinary pain treatment after distinguishing acute pain from chronic pain and evaluating various facets of a patient's pain. A multidisciplinary approach has been applied in Europe and the USA since Bonica $^{16}$ highlighted the necessity of such an approach in the 1950s. ${ }^{17}$ Multidisciplinary approaches are delivered by a team of professionals and may include education for patients, patient-centered cognitive behavioral therapy, exercise therapy, and pain-coping training. Professional groups involved include doctors from various medical departments (eg orthopedic surgeons, psychiatrists, anesthesiologists, physicians, neurologists, dentists), nurses, physical therapists, occupational therapists, clinical psychologists, pharmacists, nutritionists, and social workers. Each professional considers the patient's pathology after open discussions in conference, and contributes to a comprehensive plan that focuses on the patient's life, including sleep and nutritional support.

\section{Characteristics of our inpatient multidisciplinary pain management program}

An innovation of our multidisciplinary pain management program was the inpatient component. ${ }^{3}$ 


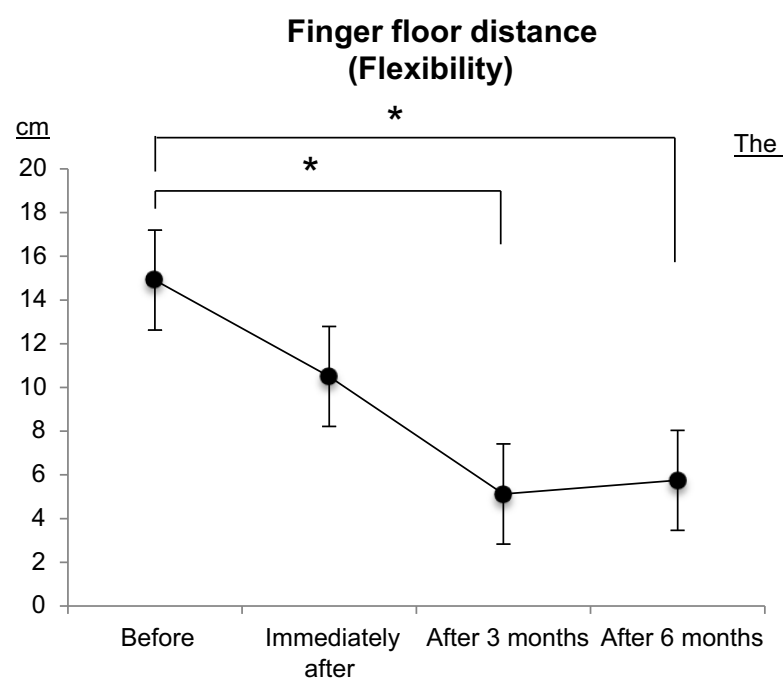

30-second sit to stand test

(Muscle endurance)

The number of times

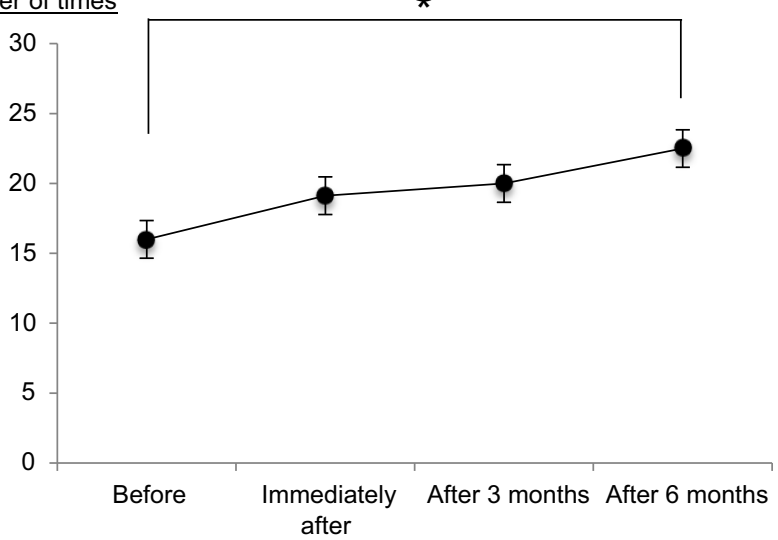

6 minutes walking

2-step test

Points (Walking ability)

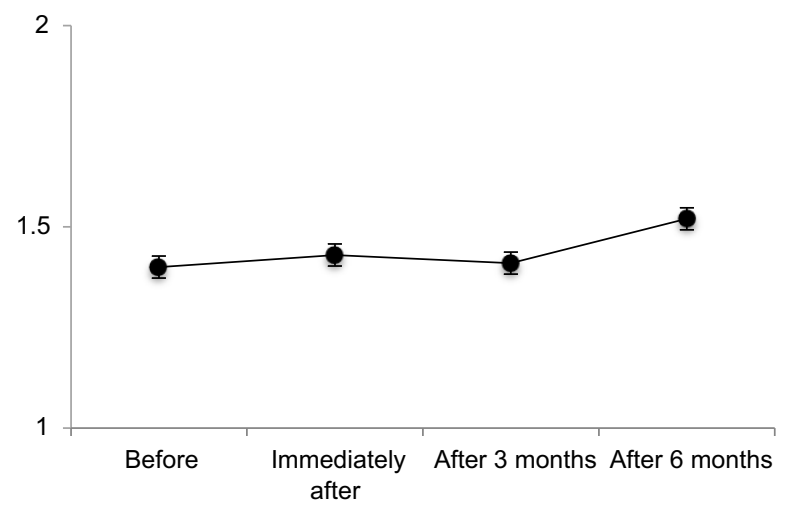

(Physical fitness)

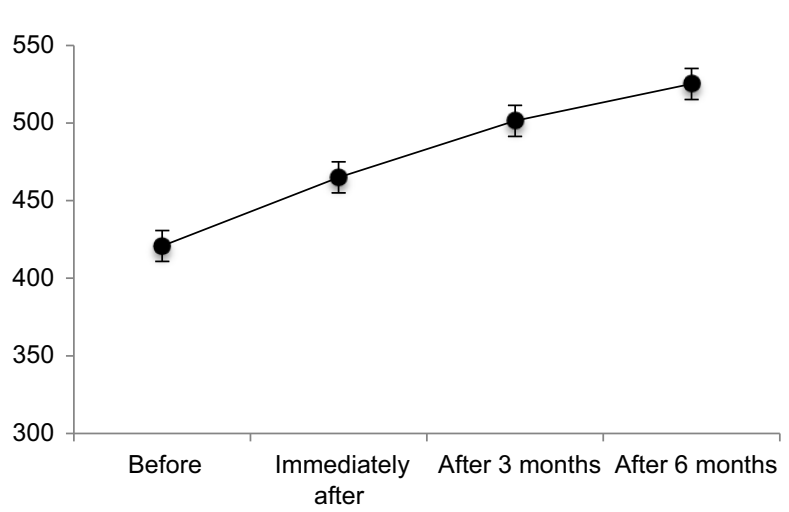

Figure 3 Changes in physical ability. Statistically significant improvement was seen in finger-floor distance (FFD) (flexibility) at $3(p=0.04)$ and $6(p=0.04)$ months after the program, and the 30-second sit-to-stand test (muscle endurance) at 6 months $(p=0.012)$. ${ }_{p}<0.05$.

\section{Cognitive behavioral therapy}

The recognition or perception of pain is considered as important as pain sensation. ${ }^{18}$ The essence of cognitive behavioral therapy in pain control is teaching perspectives and methods that help to improve pain self-management (particularly for problems related to external accidental stress), and develop new behaviors via operant conditioning that will improve social skills learning. In our facility, we classified patients into three subgroups (DYS-type, IDtype, and AC-type) using the WHYMPI. ${ }^{8}$ The subgroups were assigned during initial pain psychology interviews, and pain self-management was taught depending on subgroup assignment (type of pain). DYS-type patients can be effectively managed through operant conditioning, and patients in this group were rewarded if they attained the goals we set. For patients categorized as ID-type, it was important to help their families understand the concept of chronic musculoskeletal pain and how to change their way of attending to the patient. As patients in the AC-type subgroup tend to have difficulty adapting to some situations, we prioritized teaching this group about pacing during activities through exercise. We supervised all treatment for patients and family members. Our program aimed to help patients escape from passivity, dependency, and the tendency to be controlled by their external environment, so that they were able to manage their pain by themselves. The treatment goal was improving patients' QOL despite their chronic pain.

\section{Exercise therapy}

Exercise and stretching with pacing improves flexibility, muscle endurance, walking ability, and physical fitness. It also activates dopaminergic/serotonergic and norepinephrinergic 
neurotransmission in the descending spinal pain-inhibition pathways. ${ }^{19-22}$ Changes in whole locomotorium, such as muscle atrophy, muscle degeneration, shortening of connective tissue, osteoporosis, and psychosocial factors are associated with increased chronic musculoskeletal pain. In turn, chronic musculoskeletal pain leads to disuse of the locomotorium to avoid pain. Some cases fall into kinesiophobia, where patients tend not to move their body because of the fear of pain worsening. Pain typically increases when moving in disuse conditions, meaning the fear of moving may be reinforced. Moreover, patients may develop avoidance reactions and excess wariness. We can explain these states using a fearavoidance model. ${ }^{18,23}$ The goal of exercise therapy in our program was to allow patients to escape from this type of negative feedback loop, improve their flexibility and physical strength, promote healthy living and improved QOL, and support the recovery of physical ability. Exercise therapy can be effective on its own; however, the effects are greater if it is combined with cognitive behavioral therapy.

\section{Outpatient management after inpatient multidisciplinary/interdisciplinary pain management programs for chronic musculoskeletal pain in other facilities in worldwide}

Several studies have reported on follow-up after inpatient multidisciplinary or interdisciplinary pain management programs in other countries. ${ }^{24-33}$ We compared the outcomes from our program with those from other facilities. Table 4 shows the follow-up outcomes after inpatient programs in other countries. $^{24-33}$ The Pain Treatment Center at the Red Cross Hospital in Kassel, Germany ${ }^{24}$ offers an inpatient multidisciplinary program for neuropathic pain, delivered by a team of neurologists, physiotherapists, occupational therapists, psychotherapists, and social workers. Patients are followed for 3 months after the program (Table 4). The HRC Bethesda Children's Hospital in Budapest, Hungary ${ }^{25}$ has a 2-week inpatient interdisciplinary program for severe chronic pediatric pain. The treatment team comprises pediatric psychiatrists, clinical psychotherapist/family therapists, psychologists, physiotherapists, occupational therapists, social workers, pediatric anesthesiologists, pediatric nurses, and pediatrician/family therapists. Patients are followed for 2 years (Table 4). In Tampa, Florida (USA), the James A. Haley Veterans' Hospital $^{26}$ has a 3-week inpatient interdisciplinary chronic pain rehabilitation program for patients with chronic pain syndrome (non-cancer pain). The program delivery team includes doctors, nurses, physiotherapists, occupational therapists, recreational therapists, kinesiotherapists, vocational rehabilitation therapists, pharmacists, social workers, and a chaplain. Patients are followed for 3 months (Table 4). That program is similar to our program in length (3 weeks) and collaboration with patients' families.

Lillehammer Hospital for Rheumatic Diseases in Norway $^{27}$ offers a 1-week inpatient multidisciplinary selfmanagement program for patients with fibromyalgia. That program involves a team of rheumatologists, physiotherapists, occupational therapists, nurses, dieticians, and social workers. Patients are followed for 3 weeks after the program. The program had no effect on psychological distress, functional and symptomatic consequences, and self-efficacy for patients with fibromyalgia; however, there was a small short-term effect on skills and behavior important for managing and participating in healthcare (Table 4). The Pain Centre of the University Medical Centre Groningen in the Netherlands ${ }^{28}$ offers 4-week inpatient multidisciplinary cognitive behavioral treatment for patients with chronic pain. Treatment is delivered by a team including neurologists, physicians, physical therapists, and psychologists. Patients are followed for 1 year (Table 4). The Department of Rheumatology and Physical Medicine, University Hospital of Zurich, Switzerland ${ }^{29}$ offers a 4-week inpatient interdisciplinary pain program for patients with chronic pain, which involves a team of physicians, physical therapists, psychiatrists, and psychologists. ${ }^{30,31}$ That program showed a strong association between change in pain severity and physical functioning and change in baseline affective health and coping levels during the first outpatient management period. The program follows patients until 5months after discharge (Table 4). A 4-week inpatient cognitive behavioral pain program for patients with chronic pain is offered at INPUT Pain Management, St Thomas' Hospital, London (UK). ${ }^{32}$ That program involves a team psychologists, physiotherapists, nurses, occupational therapists, and anesthetists. Patients are followed for 6 months. Although that program has similarities to ours, it differs in length (4 weeks) and does not include nutritionists (nutritional education), clinical psychologists, or pharmacists. The program outcomes (QOL, pain intensity, psychological function, and physical function) were also similar to our results immediately after program, and were well maintained at the 6-month follow-up (Table 4). Finally, an inpatient program (at least 12 days of treatment) is offered for patients with chronic spinal back pain at the Loreley Hospital of Conservative Orthopaedics and Center for 


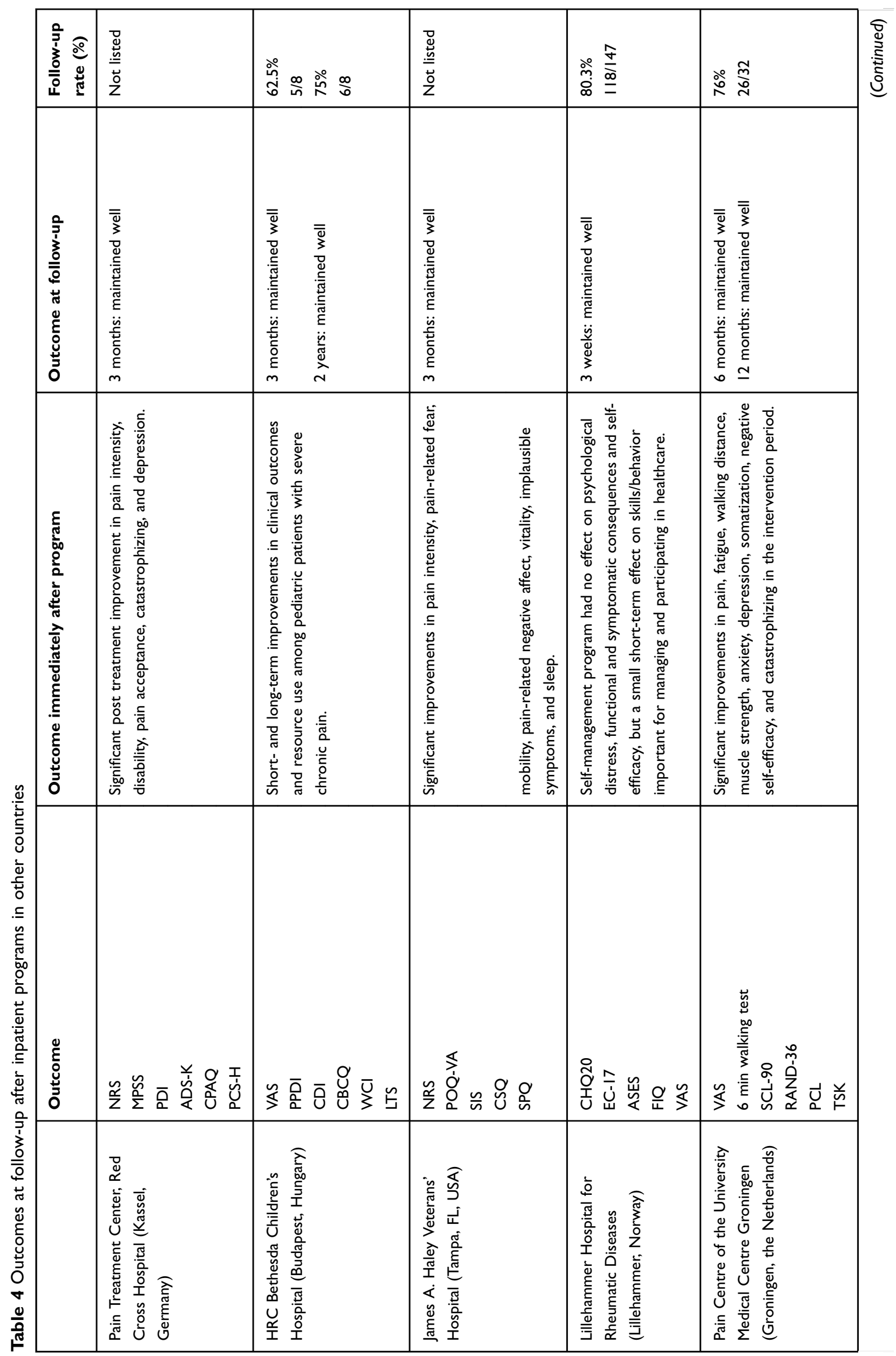




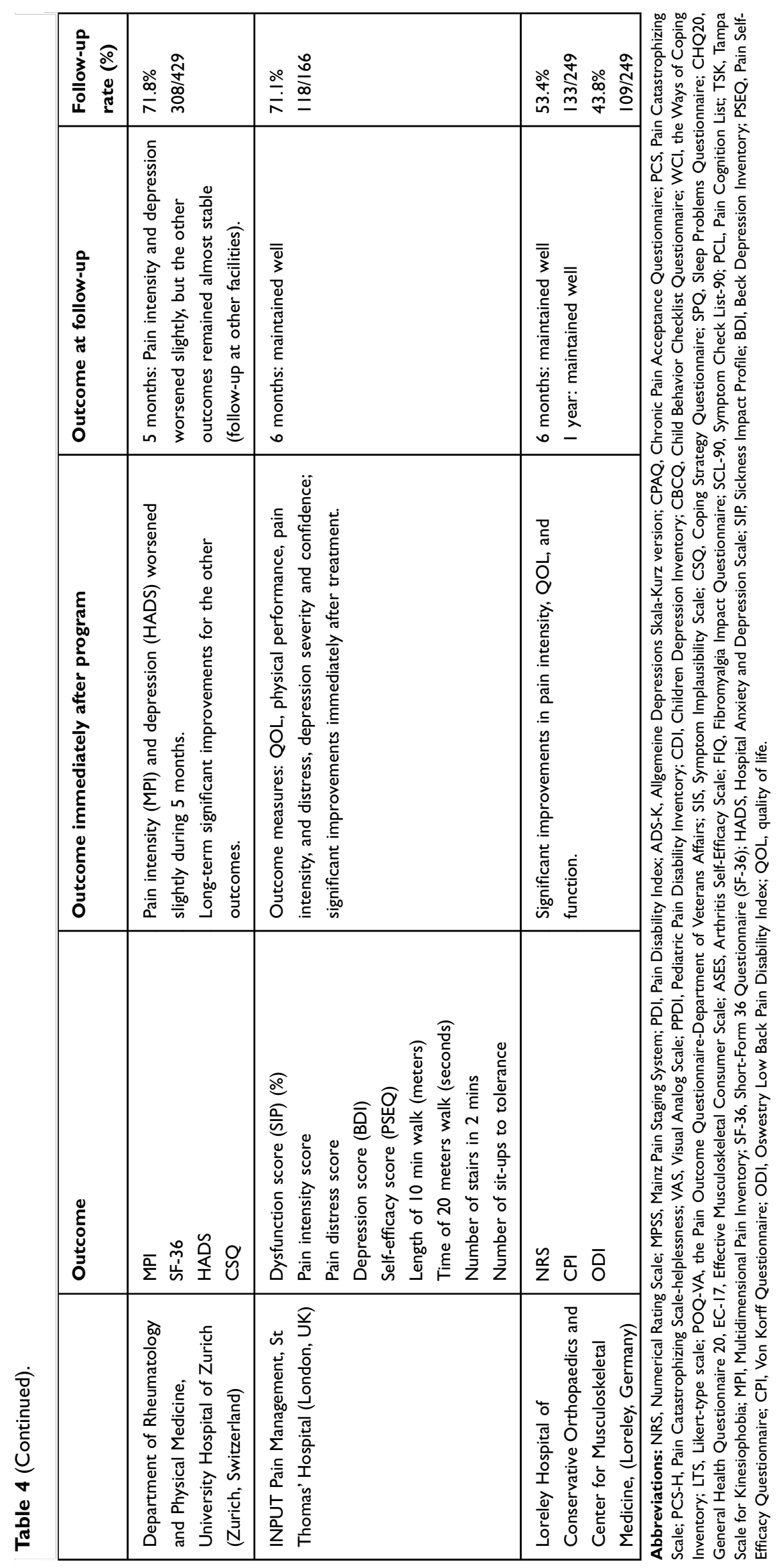


Musculoskeletal Medicine, Germany. ${ }^{33}$ Patients are followed for 12 months. That program is similar to ours, but differs in that a constant period is not used for all patients, there is no nutritional education, and the team does not include clinical psychologists, pharmacists, or nutritionists.

Overall, the outcomes of our inpatient multidisciplinary pain management program were consistent with similar programs in other countries ${ }^{24-33}$ regarding improved pain intensity, psychosocial factors (eg fear-avoidance), physical function, and QOL during the follow-up period.

\section{Limitations}

\section{Controversial points of our program}

In addition to our program's advantages, it also had a few weak or controversial points. First, although our program is open to all, we need to carefully consider each patient's rehabilitation. Second, because it may be difficult to sufficiently change how patients think and behave in a short period, patients may need to be followed-up after discharge. Finally, a large number of medical personnel are involved in our inpatient program, and their incomes may not be adequate given the insurance system for chronic pain in Japan. These points need to be addressed before our inpatient program can be generalized.

\section{Limitations of this study}

The present study had certain important limitations. First, there was no control group. We compared the treatment outcomes of our program with similar programs in other countries; however, further studies including control groups are required. Second, our study population was small, and larger populations are needed in subsequent studies. Third, the follow-up period was relatively short, and more studies with long-term follow-up are needed to evaluate long-term outcomes.

\section{Conclusion}

Our inpatient pain management program may be able to improve patients' ability to cope with their chronic musculoskeletal pain, as well as their QOL and physical abilities. Our program is currently being expanded to better assist patients with chronic musculoskeletal pain, from immediately after the program to 6-month follow-up.

\section{Abbreviations}

QOL, quality of life; IASP, the International Association for the Study of Pain; DYS, dysfunctional; ID, interpersonally distressed; AC, adaptive coper; WHYMPI, West Haven-Yale Multidimensional Pain Inventory; BPI, Brief Pain Inventory; PCS, Pain Catastrophizing Scale; PDAS, Pain Disability Assessment Scale; HADS, Hospital Anxiety and Depression Scale; PSEQ, Pain Self-Efficacy Questionnaire; EQ-5D, EuroQol five dimensions questionnaire.

\section{Acknowledgments}

The authors are grateful to all participants in this study and the clinical staff who supported this research: Koh Matsudaira, Akira Onda, Hayato Shimazaki, Hidemi Ohsuka, Saori Watanabe, Kana Hongo, Yukie Moto, Mami Tanimoto, Kenji Nihei, Minoru Iwasaki, Kazuaki Suzuki, Miho Ohuchi, Yuki Haruyama, Takahiro Yatsuda, Yoko Arase, Aki Kiryu, Yurika Saito, Yuki Ando, Megumi Sato, Yukiko Kokubun, Tomoko Fukuchi, Shiori Suganuma, and Mika Kanazawa. We thank Audrey Holmes, MA, from Edanz Group for editing a draft of this manuscript.

\section{Author contributions}

All authors made substantial contributions to conception and design, acquisition of data, or analysis and interpretation of data, took part in drafting the article or revising it critically for important intellectual content, gave final approval of the version to be published, and agree to be accountable for all aspects of the work.

\section{Disclosure}

The authors report no conflicts of interest in this work.

\section{References}

1. Turk DC, Monarch ES. Biopsychosocial perspective on chronic pain. In: Turk DC, Gatchel RJ, editors. Psychological Approaches to Pain Management: A Practitioner's Handbook. New York: Guilford press; 2002.

2. Takahashi N, Kasahara S, Yabuki S. Development and implementation of an inpatient multidisciplinary pain management program for patients with intractable chronic musculoskeletal pain in Japan: preliminary report. J Pain Res. 2018;1:201-211. doi:10.2147/JPR. S154171

3. Flor H, Turk DC. Chronic Pain: an Integrated Biobehavioral Approach. Washington DC: IASP Press; 2011.

4. Loeser JD, Butler SH, Chapman CR, Turk DC. Multidisciplinary pain management. In: Loeser JD, editor. Bonica's Management of Pain. Philadelphia: Lippincott Williams and Wilkins; 2001.

5. Turk DC, Okifuji A. Multidisciplinary pain management. In: Loeser JD, editor. Bonica's Management of Pain. Philadelphia: Lippincott Williams and Wilkins; 2001.

6. Kamper SJ, Apeldoorn AT, Chiarotto A, et al. Multidisciplinary biopsychosocial rehabilitation for chronic low back pain: Cochrane systematic review and meta-analysis. BMJ. 2015;350:h444. doi:10.1136/ bmj.h444 
7. Otis JD, Reid MC, Kerns RD. The management of chronic pain in the primary care setting. In: James LC, Folen RA, editors. Primary Care Clinical Health Psychology: A Model for the Next Frontier. Washington DC: American Psychological Association Press; 2005.

8. Kerns RD, Turk DC, Rudy TE. The West Haven-Yale Multidimensional Pain Inventory (WHYMPI). Pain. 1985;23 (4):345-356. doi:10.1016/0304-3959(85)90004-1

9. Ceeland CS, Ryan KM. Pain assessment: global use of brief pain inventory. Ann Acad Med Singapore. 1994;23(2):129-138.

10. Osman A, Barrios FX, Gutierrez PM, Kopper BA, Merrifield T, Grittmann L. The pain catastrophizing scale: further psychometric evaluation with adult samples. J Behav Med. 2000;23(4):351-365. doi:10.1023/A:1005548801037

11. Yamashiro K, Arimura T, Iwaki R, Jensen MP, Kubo C, Hosoi M. A multidimensional measure of pain interference: reliability and validity of the pain disability assessment scale. Clin J Pain. 2011;27 (4):38-343. doi:10.1097/AJP.0b013e318204858a

12. Zigmond AS, Snaith RP. Acta psychiatrica scandinavica. Hosp Anxiety Depression Scale. 1983;67(6):361-370.

13. Nicholas MK. The pain self-efficacy questionnaire: taking pain into account. Eur J Pain. 2007;11(2):153-163. doi:10.1016/j.ejpain.2005. 12.008

14. EuroQOL Group. EuroQol - a new facility for the measurement of health-related quality of life. Health Policy. 1990;16(3):199-208. doi:10.1016/0168-8510(90)90421-9

15. Chiarotto A, Vanti C, Cedraschi C, et al. Responsiveness and minimal important change of the pain self-efficacy questionnaire and short forms in patients with chronic low back pain. J Pain. 2016;17 (6):707-718. doi:10.1016/j.jpain.2015.12.006

16. Bonica JJ. Management of intractable pain in general practice. GP. 1996;33(1):107-123.

17. Scascighini L, Toma V, Dober-Spielmann S, Sprott H. Multidisciplinary treatment for chronic pain: a systematic review of interventions and outcomes. Rheumatology (Oxford). 2008;47 (5):670-678. doi:10.1093/rheumatology/ken021

18. Turk DC, Flor H. Chronic pain: a biobehavioral perspective. In: Gatchel RJ, Turk DC, editors. Psychosocial Factors in : Critical PerspectivesPain. New York:The Guilford Press; 1999:18-34.

19. Finan PH, Smith MT. The comorbidity of insomnia, chronic pain, and depression: dopamine as a putative mechanism. Sleep Med Rev. 2013;17(3):173-183. doi:10.1016/j.smrv.2012.03.003

20. Ossipov MH, Morimura K, Porreca F. Descending pain modulation and chronification of pain. Curr Opin Support Care. 2014;8(2):143-151.

21. Zheng X, Hasegawa H. Central dopaminergic neurotransmission plays an important role in thermoregulation and performance during endurance exercise. Eur J Sport Sci. 2016;16(7):818-828. doi:10.1080/17461391.2015.1111938

22. Cordeiro LMS, Rabelo PCR, Moraes MM, et al. Physical exerciseinduced fatigue: the role of serotonergic and dopaminergic systems. Braz J Med Biol Res. 2017;50(12):e6432. doi:10.1590/1414431X20176432

Journal of Pain Research

\section{Publish your work in this journal}

The Journal of Pain Research is an international, peer reviewed, open access, online journal that welcomes laboratory and clinical findings in the fields of pain research and the prevention and management of pain. Original research, reviews, symposium reports, hypothesis formation and commentaries are all considered for publication. The manuscript

Submit your manuscript here: https://www.dovepress.com/journal-of-pain-research-journal
23. Leeuw M, Goossens ME, Linton SJ, Crombez G, Boersma K, Vlaeyen JW. The fear-avoidance model of musculoskeletal pain: current state of scientific evidence. J Behav Med. 2006;30(1):77-94. doi:10.1007/s10865-006-9081-4

24. Shaygan M, Böger A, Kröner-Herwig B. Predicting factors of outcome in multidisciplinary treatment of chronic neuropathic pain. $J$ Pain Res. 2018;11:2433-2443. doi:10.2147/JPR.S175817

25. Major J, Varga ZK, Gyimesi-Szikszai A, Ádám S. A two-week inpatient programme with a booster improved long-term management of severe chronic paediatric pain. J Child Health Care. 2017;21 (2):171-180. doi:10.1177/1367493517697479

26. Murphy JL, Phillips KM, Rafie S. Sex differences between veterans participating in interdisciplinary chronic pain rehabilitation. J Rehabil Rev Dev. 2016;53(1):83-94. doi:10.1682/JRRD.2014.10.0250

27. Hamnes B, Mowinckel P, Kjeken I, Hagen K. Effects of a one week multidisciplinary inpatient self-management programme for patients with fibromyalgia: a randomized controlled trail. $B M C$ Musculoskeletal Disord. 2012;13:189. doi:10.1186/1471-2474-13189

28. van Wilgen CP, Dijkstra PU, Versteegen GJ, Fleuren MJ, Stewart R, van Wijhe M. Chronic pain and severe disuse syndrome: long-term outcome of an inpatient multidisciplinary cognitive behavioural programme. $J$ Rehabil Med. 2009;41(3):122-128. doi:10.2340/ 16501977-0292

29. Fuss I, Angst F, Lehmann S, Michel BA, Aeschlimann A. Prognostic factors for pain relief and functional improvement in chronic pain after inpatient rehabilitation. Clin J Pain. 2014;30(4):279-285. doi:10.1097/AJP.0b013e31829a4d11

30. Angst F, Brioschi R, Main CJ, Lehmann S, Aeschlimann A. Interdisciplinary rehabilitation in fibromyalgia and chronic back pain: a prospective outcome study. J Pain. 2006;7(11):807-815. doi:10.1016/j.jpain.2006.03.009

31. Angst F, Verra ML, Lehmann S, Brioschi R, Aeschlimann A. Clinical effectiveness of an interdisciplinary pain management programme compared with standard inpatient rehabilitation in chronic pain: a naturalistic, prospective controlled cohort study. $J$ Rehabil Med. 2009;41(7):569-575. doi:10.2340/16501977-0381

32. Williams AC, Nicholas PH, Richardson PH, et al. Evaluation of a cognitive behavioural programme for rehabilitating patients with chronic pain. Br J Gen Pract. 1993;43(377):513-518.

33. Steinmetz A, Psczolla M, Seidel W, Niemier K, Derlien S, Nisser J. Effect of subgroup-specific multimodal therapy on chronic spinal back pain and function - a prospective inpatient multicentre clinical trial in Germany. Medicine. 2019;98(1):e13825. doi:10.1097/ MD.0000000000013825 management system is completely online and includes a very quick and fair peer-review system, which is all easy to use. Visit http:// www.dovepress.com/testimonials.php to read real quotes from published authors. 